\title{
SPHERICAL DESIGNS, DISCREPANCY AND NUMERICAL INTEGRATION
}

\author{
PETER J. GRABNER AND ROBERT F. TICHY
}

\begin{abstract}
A spherical design is a point configuration on the sphere, which yields exact equal-weight quadrature formulae for polynomials up to a given degree. Until now only very specific constructions for spherical designs are known. We establish connections to spherical cap discrepancy and show some general discrepancy bounds. Furthermore, we reformulate the problem of constructing designs as an optimization problem and develop an algorithm for finding 'practical designs'.
\end{abstract}

\section{INTRODUCTION}

Numerical integration is an important problem in applied mathematics. There are several possibilities for measuring the quality of an approximate integration method. As an example, let us first consider the case of the interval $U=[-1,1]$ with quadrature points $x_{1}, \ldots, x_{N}$. Chebyshev computed the set of quadrature points such that for all polynomials $p(x)$ of degree $t \leq 7$ the equality

$$
\frac{1}{t} \sum_{n=1}^{t} p\left(x_{n}\right)=\frac{1}{2} \int_{-1}^{1} p(x) d x
$$

holds. Radau computed the quadrature points for $t=9$ and found that for $t=8$ some quadrature points are complex. Bernstein proved the surprising result, that for $t>9$ there is no such formula with $t$ real quadrature points (cf. [14]). Note that all weights are equal, otherwise by Gauss integration it is possible to integrate polynomials of degree $2 t-1$ by $t$ points. The following natural question arises: How many distinct quadrature points are necessary to integrate all polynomials of degree $\leq t$ ? In the recent paper [19], G. Wagner proved the upper bound $N \ll t^{22}$ for the number of quadrature points; note that Bernstein proved that $N>\frac{t^{2}}{16}$. (We use Vinogradov's notation $f \ll g$ for $|f| \leq C|g|$ for a suitable positive constant $C$.) For a more detailed survey we refer to [5].

Seymour and Zaslavsky [17] have shown in a much more general situation that for any degree $t$ there exist finitely many quadrature points which yield an exact equal-weight quadrature formula. (More precisely, they considered

Received by the editor July 16, 1991 and, in revised form, February 3, 1992.

1991 Mathematics Subject Classification. Primary 11K45, 65C05.

The authors were supported by the Austrian Science Foundation (Project Nr. P8274PHY). 
functions on topological spaces and arbitrary probability measures.) However, no effective bound for the number of these quadrature points is given. The one-dimensional result of [19], on the other hand, can be applied to construct quadrature points on the $d$-sphere which yield exact integration of harmonic polynomials up to a prescribed order.

A second method for classifying numerical integration methods is based on Koksma's inequality (cf. [9, p. 143]):

$$
\left|\frac{1}{N} \sum_{n=1}^{N} f\left(x_{n}\right)-\int_{0}^{1} f(x) d x\right| \leq V(f) D_{N}\left(x_{1}, \ldots, x_{N}\right),
$$

where $V(f)$ is the total variation of $f$ and

$$
D_{N}\left(x_{1}, \ldots, x_{N}\right)=\sup _{J}\left|\frac{1}{N} \sum_{k=1}^{N} \chi_{J}\left(x_{k}\right)-\lambda(J)\right|
$$

is the discrepancy of the finite point sequence $\left\{x_{n}\right\}$ ( $\chi_{J}$ denotes the characteristic function of the interval $J$ of length $\lambda(J))$. It can easily be seen that the discrepancy is minimal for equidistant points.

Of course, the estimate (1.2) is of no interest for numerical integration, but the concept of discrepancy is very fruitful for numerical applications in higher dimensions (cf. [10,9]). The aim of this paper is to deal with numerical integration problems on the $d$-dimensional sphere equipped with the normalized surface measure $\sigma$. As an approximation to the surface integral

$$
\int_{S^{d}} f(x) d \sigma(x)
$$

we consider the arithmetic mean

$$
\frac{1}{N} \sum_{n=1}^{N} f\left(x_{n}\right) \text {. }
$$

The quality of approximation depends on the smoothness of the function $f$ and on the distribution of the points on the sphere. As a measure for this distribution we will use the spherical cap discrepancy

$$
D_{N}^{C}\left(x_{1}, \ldots, x_{N}\right)=\sup _{C}\left|\frac{1}{N} \sum_{k=1}^{N} \chi_{C}\left(x_{k}\right)-\sigma(C)\right|,
$$

where the supremum is extended over all spherical caps $C$; a cap is defined as intersection of the sphere and a halfspace. In a series of papers [8], E. Hlawka investigated several different notions of discrepancy, for instance the so-called Lipschitz discrepancy and a concept of discrepancy based on Green's function. In the following we will only use the spherical cap discrepancy, because it seems to be the most natural concept. From the theory of irregularities of distribution (cf. [1]) the following general lower bound is known:

$$
D_{N}^{C}\left(x_{1}, \ldots, x_{N}\right) \gg \frac{1}{N^{\frac{1}{2}+\frac{1}{2 d}}},
$$

and this estimate is optimal up to a possible logarithmic factor.

Generalizing point sets satisfying (1.1) to the case of the $d$-dimensional sphere leads to the concept of spherical design. 
Definition 1. A point set $x_{1}, \ldots, x_{N} \in S^{d}$ is called a spherical $t$-design of order $N$ if

$$
\frac{1}{N} \sum_{n=1}^{N} p\left(x_{n}\right)=\int_{S^{d}} p(x) d \sigma(x)
$$

for all polynomials (in $d+1$ variables restricted to $S^{d}$ ) of degree $\leq t$.

Remark 1. Obviously, this definition is equivalent to

$$
\frac{1}{N} \sum_{n=1}^{N} K_{m, k}\left(x_{n}\right)=0
$$

for all spherical harmonics $K_{m, k}, m=1, \ldots, t$ and $k=1, \ldots, Z(d, m)$, where $Z(d, m)$ is the number of linearly independent spherical harmonics of order $m$ (cf. [13]).

For applications in numerical analysis it is necessary to know effective constructions of spherical $t$-designs of order $N$ with small $N$ and large $t$. Seymour and Zaslavsky [17] proved the existence of $t$-designs for any positive integer $t$ and gave a first rough bound for $N$ in terms of $t$. G. Wagner [19] used the investigation of the one-dimensional case to give an explicit construction for a $t$-design with $N \ll t^{8 d^{3}}$ for infinitely many $t$. Furthermore, he showed that for arbitrary $t$ and all $N \gg t^{12 d^{4}}$ there exists a spherical $t$-design of $N$ points. These bounds are of little use for practical applications and seem to be far away from reality. MacLaren [12] used finite subgroups of the rotation group to construct designs for special values of $t$. Seidel et al. $[16,3]$ considered refinements of this method, and recently Godsil [6] extended this group-theoretical approach to a more general situation. Since there are only finitely many polyhedral groups it seems to be impossible to construct spherical $t$-designs for arbitrarily large $t$ by this method.

The aim of this paper is to establish a connection between discrepancy and spherical designs. We will give effective constructions for low-discrepancy point sets on the $d$-dimensional sphere. Furthermore the construction problem of spherical designs is reformulated as an optimization problem which can be solved by computational methods.

\section{NUMERICAL INTEGRATION AND DISCREPANCY}

Generalizing the well-known Erdös-Turán inequality, one of us [7] proved the following bound for the spherical cap discrepancy: Let $x_{n}, n=1, \ldots, N$, be a finite point sequence in $S^{d}$; then for an arbitrary positive integer $M$ the following inequality holds

$$
\begin{aligned}
& D_{N}^{C}\left(x_{1}, \ldots, x_{N}\right) \\
& \quad \leq \frac{c_{1}(d)}{M+1}+\sum_{k=1}^{M}\left(\frac{c_{2}(d)}{k}+\frac{c_{3}(d)}{M+1}\right) \sum_{j=1}^{Z(d, k)}\left|\frac{1}{N} \sum_{n=1}^{N} K_{k, j}\left(x_{n}\right)\right|,
\end{aligned}
$$


where the constants can be taken to be

$$
\begin{aligned}
& c_{1}(d)=\max \left(1, \frac{\pi}{c_{d}}-1\right)+\frac{\pi}{c_{d}}, \\
& c_{2}(d)=\frac{1}{\pi} \sqrt{\omega_{d}} \Gamma\left(\frac{d+1}{2}\right) 2^{d+1} e^{d}, \\
& c_{3}(d)=\sqrt{\omega_{d}} e^{d-1} 2^{\frac{d+3}{2}} \Gamma\left(\frac{d+1}{2}\right),
\end{aligned}
$$

with $c_{d}=\frac{\sqrt{\pi \Gamma\left(\frac{d}{2}\right)}}{\Gamma\left(\frac{d+1}{2}\right)}, \omega_{d}=\frac{2 \pi^{\frac{d+1}{2}}}{\Gamma\left(\frac{d+1}{2}\right)}$.

Now suppose that there exists a $t$-design with $N=c t^{L}$ points $x_{1}, \ldots, x_{N}$. Inserting these points in (2.1) and setting $M=t$, we immediately obtain by the definition of a $t$-design that

$$
D_{N}^{C}\left(x_{1}, \ldots, x_{N}\right) \ll \frac{1}{N^{\frac{1}{L}}} .
$$

The way in which (2.2) follows from (2.1) mirrors a similar phenomenon in the theory of good lattice points (cf. [9, Chapter 4]). From [3] we know that $L \geq d$. Thus, our bound (2.2) is not optimal (cf. (1.6)). However, it should be remarked that Beck's approach (cf. [1, Theorem 24D, p.182]) makes use of probabilistic arguments and yields the existence (without effective construction) of spherical sequences $\left\{x_{n}\right\}$ with discrepancy

$$
D_{N}^{C}\left(x_{1}, \ldots, x_{N}\right) \ll \frac{\log ^{\frac{1}{2}} N}{N^{\frac{1}{2}+\frac{1}{2 d}}} .
$$

In the case of the two-dimensional sphere, Lubotzky, Phillips, and Sarnak [11] established a group-theoretical construction of a point set $x_{1}, \ldots, x_{N}$ satisfying $D_{N}^{C}\left(x_{1}, \ldots, x_{N}\right) \ll N^{-\frac{1}{3}}$. The main ingredient of their proof is the deep fact that the group generated by the rotations $A, B, C$ with angle $\arccos \left(-\frac{3}{5}\right)$ around the coordinate axes is free. The approach is essentially restricted to $d=2$.

By (2.1), the spherical cap discrepancy can be estimated in terms of spherical harmonics. In the following we will establish a bound for the approximation error in terms of spherical harmonics.

Theorem 1. Let $f$ be a continuous function on $S^{d}$ satisfying

$$
|f(x)-f(y)| \leq C_{f} \arccos (\langle x, y\rangle),
$$

and let $V(f)=\sup _{x, y}|f(x)-f(y)|$. Then for all positive integers $M$,

$$
\left|\frac{1}{N} \sum_{n=1}^{N} f\left(x_{n}\right)-\int_{S^{d}} f(x) d \sigma(x)\right| \leq 6 C_{f} \frac{d}{M}+V(f) \sum_{m=1}^{2 M} \sum_{l=1}^{Z(d, m)}\left|\frac{1}{N} \sum_{n=1}^{N} K_{m, l}\left(x_{n}\right)\right| .
$$

Proof. Let

$$
K_{M}(t)=a_{M}\left(\frac{P_{M+1}^{d}(t)}{t-\nu_{M+1}}\right)^{2}
$$

where $P_{M+1}^{d}$ is the ultraspherical polynomial with parameter $\lambda=\frac{d-2}{2}$ of degree $M+1, \nu_{M+1}$ is the largest zero of $P_{M+1}^{d}$ and $a_{M}$ is chosen such that

$$
\int_{S^{d}} K_{M}(\langle x, y\rangle) d \sigma(x)=1, \quad y \in S^{d}
$$


This approximation kernel is due to Newman and Shapiro [15]. Let furthermore

$$
f_{M}(x)=\int_{S^{d}} f(y) K_{M}(\langle x, y\rangle) d \sigma(y) .
$$

Then by using the hypotheses on $f$ we obtain

$$
\begin{aligned}
\left|f(x)-f_{M}(x)\right| & \leq \int_{S^{d}}|f(y)-f(x)| K_{M}(\langle x, y\rangle) d \sigma(y) \\
& \leq C_{f} \int_{S^{d}} \arccos \langle x, y\rangle K_{M}(\langle x, y\rangle) d \sigma(y) \\
& \leq 2 C_{f} \int_{S^{d}} \sqrt{1-\langle x, y\rangle} K_{M}(\langle x, y\rangle) d \sigma(y) \\
& \leq 2 C_{f}\left(\int_{S^{d}}(1-\langle x, y\rangle) K_{M}(\langle x, y\rangle) d \sigma(y)\right)^{1 / 2} \\
& =2 C_{f}\left(1-\frac{\int_{0}^{\pi} \cos \varphi K_{M}(\cos \varphi)(\sin \varphi)^{d-1} d \varphi}{\int_{0}^{\pi}(\sin \varphi)^{d-1} d \varphi}\right)^{1 / 2} .
\end{aligned}
$$

The quotient of integrals in the last line equals $\nu_{M+1}$; by results in Szegö [18, $\S 6.21]$ and Newman and Shapiro [15], $\nu_{M+1} \geq 1-5 \frac{d^{2}}{M^{2}}$. Therefore, the estimate

holds.

$$
\left|f(x)-f_{M}(x)\right| \leq 6 C_{f} \frac{d}{M}
$$

We now use this approximation to estimate the error of our numerical integration method:

$$
\begin{aligned}
& \left|\frac{1}{N} \sum_{n=1}^{N} f\left(x_{n}\right)-\int_{S^{d}} f(x) d \sigma(x)\right| \\
& \quad \leq \frac{1}{N} \sum_{n=1}^{N}\left|f(x)-f_{M}(x)\right|+\frac{1}{N}\left|\sum_{n=1}^{N} f_{M}\left(x_{n}\right)-\int_{S^{d}} f(x) d \sigma(x)\right| \\
& \quad \leq 6 C_{f} \frac{d}{M}+\sum_{m=1}^{2 M} \sum_{l=1}^{Z(d, m)}|\hat{f}(m, l)|\left|\frac{1}{N} \sum_{n=1}^{N} K_{m, l}\left(x_{n}\right)\right|,
\end{aligned}
$$

where $\hat{f}(m, l)=\int_{S^{d}} f(x) K_{m, l}(x) d \sigma(x)$. For establishing the last inequality, we note that the kernel $K_{M}$ is positive and hence all coefficients in its spherical harmonic expansion are $\leq 1$. Now we use

$$
\left|f(x)-\int_{S^{d}} f(x) d \sigma(x)\right| \leq V(f)
$$

and Bessel's inequality to obtain the upper bound

$$
6 C_{f} \frac{d}{M}+V(f) \sum_{m=1}^{2 M} \sum_{l=1}^{Z(d, m)}\left|\frac{1}{N} \sum_{n=1}^{N} K_{m, l}\left(x_{n}\right)\right| .
$$

Remark 2. Inserting a spherical $t$-design with $N=C t^{L}$ leads to the error bound

$$
\left|\frac{1}{N} \sum_{n=1}^{N} f\left(x_{n}\right)-\int_{S^{d}} f(x) d \sigma(x)\right| \ll \frac{1}{N^{\frac{1}{L}}} .
$$


Remark 3. A proof similar to that above yields

$$
\begin{aligned}
& \left|\frac{1}{N} \sum_{n=1}^{N} f\left(x_{n}\right)-\int_{S^{d}} f(x) d \sigma(x)\right| \\
& \quad \leq 7 \omega\left(\frac{d}{M}\right)+V(f) \sum_{m=1}^{2 M} \sum_{k=1}^{Z(d, m)}\left|\frac{1}{N} \sum_{n=1}^{N} K_{m, k}\left(x_{n}\right)\right|,
\end{aligned}
$$

where $\omega(u)$ is a function satisfying

$$
|f(x)-f(y)| \leq \omega(\arccos \langle x, y\rangle) .
$$

\section{CONSTRUCTION OF DESIGNS by Optimization}

In [19] the first explicit construction of spherical $t$-designs for arbitrary $t$ is given. The construction works by projection into the coordinate axes and applying the solution of the one-dimensional problem as mentioned in the introduction. Furthermore, Wagner shows that for arbitrary $t$ and all $N \geq c_{d} t^{12 d^{4}}$ there exists a spherical $t$-design of $N$ points. The paper [19] contains some computational errors which, however, do not affect the correctness of the result. A detailed analysis of the proof shows that the bounds can be improved to some extent by the same methods. G. Wagner himself announced such improved bounds before he died in March 1990 in an avalanche in the Alps. For instance, in the one-dimensional case he announced the existence of a set of $N$ quadrature points satisfying (1.1) with $N \gg t^{7}$. We hope that these results will be published soon.

Clearly, it is desirable to find spherical $t$-designs of $N$ points with $N$ close to $t^{d}$. For this purpose we suggest to reformulate the problem as an optimization problem. By summing up the squares of $(1.8)$ for all $k=1, \ldots, Z(d, m)$ and using the addition theorem for spherical harmonics (cf. [13, p. 9]), we obtain

$$
\sum_{j, k=1}^{N} P_{m}^{d}\left(\left\langle x_{j}, x_{k}\right\rangle\right)=0
$$

for $m=1, \ldots, t$ as a condition for a $t$-design. Taking into account that every antipodal set (these are sets which contain with every point also its antipodal point) yields exact integration for all odd $m$, we first look for point sets with

$$
\sum_{n=1}^{N} K_{m, k}\left(x_{n}\right)=0
$$

for even $m \leq t$. Then the set $x_{1}, \ldots, x_{N},-x_{1}, \ldots,-x_{N}$ is a spherical $t$ design; the order of magnitude of $N(t)$ is not affected by this construction.

By summing up (3.1) we obtain

$$
F\left(x_{1}, \ldots, x_{N}\right)=\frac{1}{N^{2}} \sum_{j, k=1}^{N} Q_{2 M}\left(\left\langle x_{j}, x_{k}\right\rangle\right)=0,
$$

where

$$
Q_{2 M}(x)=\sum_{m=1}^{M} P_{2 m}^{d}(x)
$$


as a condition for the points $x_{1}, \ldots, x_{N}$ to be part of such a $(2 M+1)$-design. We note that this value is also the absolute minimum of the sum on the righthand side for $N$ large enough. From [17] it follows that this minimum is attained for sufficiently large $N$.

In the following we assume that $F$ attains the global minimum $C_{M, N}$. In order to derive a discrepancy bound, we make use of the following

Lemma 1. There holds

$$
D_{N}^{C}\left(x_{1}, \ldots, x_{N}\right) \leq \frac{c_{1}(d)}{M+1}+c_{2}^{\prime}(d) \sum_{m=1}^{M} m^{\frac{d-3}{2}} \frac{1}{N} \sqrt{\sum_{r, s=1}^{N} P_{m}^{d}\left(\left\langle x_{r}, x_{s}\right\rangle\right)},
$$

where $c_{1}(d)$ is the same constant as in $(2.1)$ and $c_{2}^{\prime}(d)$ is some positive constant. Proof. The proof of this inequality can be easily derived from $\S 3$ in [7, pp. 133-134]. Now let $C=\left\{y \in S^{d}:\langle x, y\rangle \geq \cos \varphi\right\}$; then

$$
\#\left\{1 \leq n \leq N: x_{n} \in C\right\}=\sum_{n=0}^{N} \chi_{[\cos \varphi, 1]}\left(\left\langle x, x_{n}\right\rangle\right) .
$$

By [7, p. 133-134] we have

$$
\begin{aligned}
\left|\frac{1}{N} \sum_{n=0}^{N} \chi_{[\cos \varphi, 1]}\left(\left\langle x, x_{n}\right\rangle\right)-\sigma(C)\right| \\
\leq \frac{c_{1}(d)}{M+1}+\sum_{m=1}^{M} \frac{1}{c_{d} m^{\frac{d-1}{2}}}\left(\frac{2^{\frac{d+1}{2}} C(d+2)}{d m}+\frac{2 \pi C(d)}{M+1}\right) \omega_{d} \\
\quad \times\left|\sum_{j=1}^{Z(d, m)} K_{m, j}(x) \frac{1}{N} \sum_{n=1}^{N} K_{m, j}\left(x_{n}\right)\right|,
\end{aligned}
$$

where $C(d)=\Gamma\left(\frac{d}{2}\right) 2^{\frac{d+1}{2}} e^{\frac{d-2}{2}} / \sqrt{\pi}$ and the other constants are defined after $(2.1)$. Applying the Cauchy-Schwarz inequality for estimating the summation over $j$, and using the addition theorem for spherical harmonics (cf. [13, p. 9]) yields Lemma 1.

Inserting the minimal value $C_{M, N}$ in (3.3), summing up the powers of $m$ and using the antipodal construction, yields

$$
D_{2 N}^{C}\left( \pm x_{1}, \ldots, \pm x_{N}\right) \leq \frac{c_{1}(d)}{2 M+1}+c_{2}^{\prime \prime}(d) M^{\frac{d-1}{2}} \sqrt{C_{M, N}}
$$

with some new constant $c_{2}^{\prime \prime}(d)>0$.

Theorem 2. Let $x_{1}, \ldots, x_{N}$ be a point set such that

$$
F\left(x_{1}, \ldots, x_{N}\right)=\frac{1}{N^{2}} \sum_{r, s=1}^{N} Q_{2 M}\left(\left\langle x_{r}, x_{s}\right\rangle\right)
$$

defined in (3.2) attains its minimum value at this point set. Then the discrepancy of the antipodal set $\pm x_{1}, \ldots, \pm x_{N}$ is bounded by

$$
D_{2 N}^{C}\left( \pm x_{1}, \ldots, \pm x_{N}\right) \ll \frac{1}{N^{\frac{1}{d+2}}} .
$$


Proof. An easy computation shows

$$
\int_{S^{d}} \cdots \int_{S^{d}} F\left(x_{1}, \ldots, x_{N}\right) d \sigma\left(x_{1}\right) \cdots d \sigma\left(x_{N}\right)=\frac{M}{N} .
$$

Thus the minimal value $C_{M, N}$ is less than $\frac{M}{N}$. Inserting this in (3.4) and setting $M=\left[N^{\frac{1}{d+2}}\right]$ yields the estimate (3.5).

\section{Computational Results}

In this final section we discuss computational methods for constructing spherical point distributions in the case of the 2-sphere. The naive method for constructing a spherical design is to find an explicit solution for the system of equations (1.8). These are polynomial equations of high order and in many variables. Thus, the standard methods of computational algebra (cf. [2]) seem to be overtaxed.

McLaren [12] suggested the following method for constructing uniformly distributed point sets: Start with an icosahedron with center $M$ and fix one vertex point $P$. Consider the five edges through $P$ and fix their midpoints $P_{1}, \ldots, P_{5}$. Then the icosahedron is rotated around the axes $M P_{i}, i=$ $1, \ldots, 5$, with angle $\frac{\pi}{2}$. Thus, five new icosahedra are generated, and this procedure can be iterated. In this way a spherical point distribution is generated (points occurring repeatedly are taken only once). This algorithm can be easily implemented on computers and a MODULA- 2 code is available from the authors. Our program also produces a graphical representation (two normal projections) of the point distribution. In the case of five iterations (46860 points), Figure 1 shows the result. In the following we discuss some computations for the construction of designs on the 2-sphere by optimization. As in $\S 3$, one can show that the antipodal set $\pm x_{1}, \ldots, \pm x_{N}$ generates a $(2 M+1)$-design if the function

$$
G\left(x_{1}, \ldots, x_{N}\right)=\sum_{j, k=1}^{N}\left\langle x_{j}, x_{k}\right\rangle^{2 M}
$$

attains its minimum in the point set $x_{1}, \ldots, x_{N}$ and the value of the minimum is $\frac{N^{2}}{2 M+1}$. Note that $x^{2 M}$ is a positive linear combination of Legendre polynomials.

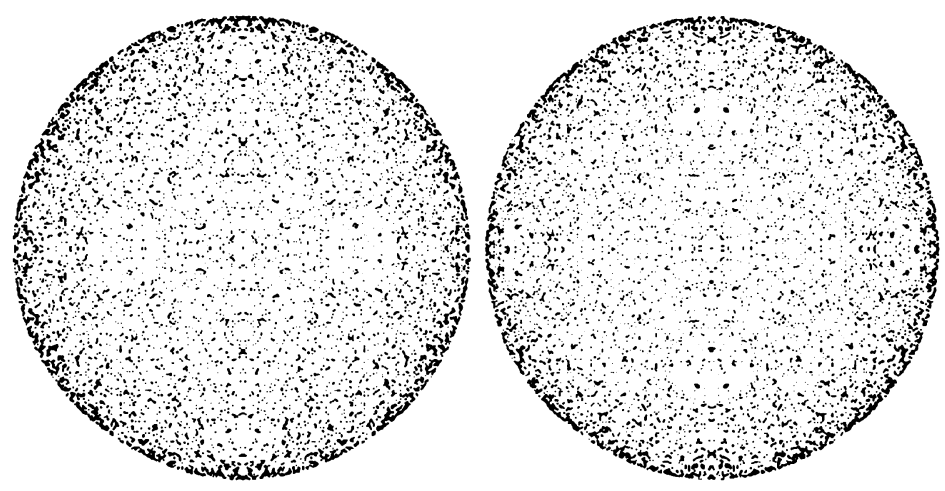

FIGURE 1 
TABLE 1

\begin{tabular}{|ll|r|r|}
\hline $\mathrm{M}$ & $\mathrm{N}$ & computed value & \multicolumn{1}{|c|}{$\frac{N^{2}}{2 M+1}$} \\
\hline 2 & 6 & & \\
3 & 11 & 17.3125 & 17.2000 \\
3 & 12 & 20.6250 & 20.5714 \\
4 & 15 & 25.2124 & 25.0000 \\
4 & 16 & 28.4768 & 28.4444 \\
5 & 21 & 40.2600 & 40.0909 \\
5 & 22 & 44.0767 & 44.0000 \\
6 & 33 & 83.8443 & 83.7692 \\
6 & 34 & 88.9990 & 88.9230 \\
7 & 41 & 112.1665 & 112.0666 \\
7 & 42 & 117.6981 & 117.6000 \\
8 & 54 & 171.6177 & 171.5294 \\
8 & 55 & 178.0389 & 177.9411 \\
9 & 77 & 312.2648 & 312.0526 \\
12 & 130 & 676.1551 & 676.0000 \\
14 & 180 & 1118.6311 & 1117.2413 \\
14 & 189 & 1232.7401 & 1231.7586 \\
\hline
\end{tabular}

In Table 1 we present a numerical computation of the minimum with the help of a combined stochastic and analytic procedure. The basic idea of this procedure is to determine suitable initial values by a Monte Carlo method and then improving these values by a gradient method. For more details concerning the implementation of this procedure we refer to [4]. Note that for the values $M=12$ and $M=14$ the difference between the computed value and the exact value of the minimum is within the rounding error. For smaller values of $M$ exact designs are known. We call these point configurations 'practical designs', because numerically they behave like designs but until now it is not clear whether they are $t$-designs or not. Since the computations were done on a simple PC, this method would yield practical designs of higher order by using better hardware.

\section{ACKNOWLEDGEMENT}

We are indebted to Mr. H. Fischer for doing the computational work leading to Table 1. We are grateful to two anonymous referees for their valuable suggestions improving the presentation of the paper.

\section{BIBLIOGRAPHY}

1. J. Beck and W. Chen, Irregularities of distribution, Cambridge Univ. Press, 1987.

2. B. Buchberger, Applications of Gröbner bases in non-linear computational geometry, Mathematical Aspects of Scientific Software (J.R. Rice, ed.), Springer, Berlin, 1988, pp. 59-87.

3. P. Delsarte, J. M. Goethals, and J. J. Seidel, Spherical codes and design, Geom. Dedicata 6 (1977), 363-388. 
4. H. Fischer, Beiträge zur Computerzahlentheorie: Lineare Rekursionen, Designs und Diophantische Gleichungen, Thesis, Techn. Univ. Vienna, 1992.

5. W. Gautschi, Advances in Chebyshev quadrature, Lecture Notes in Math., vol. 506, Springer, Berlin, 1976, pp. 100-121.

6. C. D. Godsil, Polynomial spaces, Discrete Math. 73 (1988/89), 71-88.

7. P. J. Grabner, Erdös-Turán type discrepancy bounds, Monatsh. Math. 111 (1991), 127-135.

8. E. Hlawka, Beiträge zur Theorie der Gleichverteilung und ihren Anwendungen I-V, Österr. Akad. Wiss. SB II 197 (1988), 1-154, 209-289.

9. L. K. Hua and Y. Wang, Application of number theory to numerical analysis, SpringerVerlag, 1981.

10. L. Kuipers and H. Niederreiter, Uniform distribution of sequences, Wiley, New York, 1974.

11. A. Lubotzky, R. Phillips, and P. Sarnak, Hecke operators and distributing points on the sphere I, II, Comm. Pure Appl. Math. 39 (1986), 149-186; 40 (1987), 401-420.

12. A.D. MacLaren, Optimal numerical integration on a sphere, Math. Comp. 17 (1963), 361383.

13. C. Müller, Spherical harmonics, Lecture Notes in Math., vol. 17 Springer-Verlag, Berlin, 1966.

14. I. P. Natanson, Konstruktive Funktionentheorie, Akademie-Verlag, Berlin, 1955.

15. D. J. Newman and H. S. Shapiro, Jackson's theorem in higher dimensions, Über Approximationstheorie (P. L. Butzer and J. Korevaar, eds.), ISNM, vol. 5, Birkhäuser-Verlag, Basel, 1964, pp. 208-219.

16. J. J. Seidel, Integration over spheres, Discrete Geometry (A. Florian, ed.), Salzburg, 1985, pp. 233-242.

17. P. D. Seymour and T. Zaslavsky, Averaging sets: A generalization of mean values and spherical designs, Adv. in Math. 52 (1984), 213-240.

18. G. Szegö, Orthogonal polynomials, Amer. Math. Soc. Colloq. Publ., vol. 23, Amer. Math. Soc., Providence, RI, 1939.

19. G. Wagner, On averaging sets, Monatsh. Math. 111 (1991), 69-78.

Institut für Mathematik, Technische Universität Graz, Steyrergasse 30, 8010 Graz, 8AUSTRIA

E-mail address: grabner@ftug.dnet.tu-graz.ac.at

E-mail address: tichy@ftug.dnet.tu-graz.ac.at 